\title{
A Novel Rat Model of Ankle Osteoarthritis Induced by the Application of Monoiodoacetate
}

Syunsuke Jimbo ${ }^{1}$, Yoshinori Terashima ${ }^{1,2^{*}}$, Tsuneo Takebayashi ${ }^{3}$, Atsushi Teramoto ${ }^{1}$, Izaya Ogon ${ }^{1}$, Kota Watanabe ${ }^{4}$, Tatsuya Sato ${ }^{2,5}$, Nobutoshi Ichise ${ }^{2}$, Noritsugu Tohse ${ }^{2}$ and Toshihiko Yamashita ${ }^{1}$

${ }^{1}$ Department of Orthopaedic Surgery, Sapporo Medical University, School of Medicine, Sapporo, Japan

${ }^{2}$ Department of Cellular Physiology and Signal Transduction, Sapporo Medical University, School of Medicine, Sapporo, Japan

${ }^{3}$ Sapporo Maruyama Orthopaedic Hospital, Sapporo, Japan

${ }^{4}$ Department of Second Division of Physical Therapy, Sapporo Medical University, School of Health Sciences, Sapporo, Japan

${ }^{5}$ Department of Cardiovascular, Renal and Metabolic Medicine, Sapporo Medical University, School of Medicine, Sapporo, Japan

*Corresponding author: Yoshinori Terashima, Department of Orthopaedic Surgery, Sapporo Medical University, School of Medicine, Sapporo, Japan, Tel: +81116112111; Fax: +81116416026, E-mail: y.terashima@sapmed.ac.jp

Received date: November 20, 2017; Accepted date: November 24, 2017; Published date: November 25, 2017

Copyright: (c) 2017 Jimbo S, et al. This is an open-access article distributed under the terms of the Creative Commons Attribution License, which permits unrestricted use, distribution, and reproduction in any medium, provided the original author and source are credited.

\begin{abstract}
Although ankle osteoarthritis is a common human joint disease, the etiological mechanisms underlying the associated pain remain unknown. We developed a new rat model of ankle osteoarthritis (OA) induced by monoiodoacetate (MIA) and evaluated the behavioral and histological characteristics of these animals. 60 male Sprague-Dawley rats were divided into three experimental groups of 20 rats. OA was induced by injecting the metabolic inhibitor MIA $(1 \mathrm{mg} / 30 \mu \mathrm{l})$ into the tibiotarsal joint of the right hind paw on two consecutive days (MIA2 group) or single day (MIA1 group). Saline was injected into sham rats using the same protocol (sham group). We examined ankle swelling, range of motion, and stride length. We also assessed pain related behavior by observing the mechanical and thermal withdrawal responses. Rats were sacrificed 28 days after MIA/saline injection, and the right ankle joints were removed. Ankle sections were stained with safranin-O fast green, and histopathological changes were observed and scored. Both MIA groups exhibited significant increase in the ankle anteroposterior and transverse diameters (i.e., swelling) on days $7,14,21$, and 28 post-injection relative to sham, as well as significantly decrease ROMs of the ankle. MIA2 rats additionally had a significantly shorter stride than did sham rats. MIA2 rats also exhibited an increased mechanical response frequency to the von Frey hair $4 \mathrm{~g}$, and $6 \mathrm{~g}$ tests, compared with sham rats, whereas MIA1 rats did not differ significantly from sham rats. Both MIA2 and MIA1 rats exhibited significantly reduced thermal latency in the hind paw relative to the sham group on day 28 post-injection. Histologically, the both MIA groups had a significantly higher score on day 28 post-injection, compared with the sham rats. MIA2 rats also exhibited more histological evidence of cartilage damage and abnormal medial tibia morphology, compared to MIA1 and sham rats.
\end{abstract}

Keywords: Ankle osteoarthritis; Monoiodoacetate (MIA); Osteoarthritis pain

\section{Introduction}

Osteoarthritis $(\mathrm{OA})$ is caused by cartilage degeneration, fibrillation, erosion, and osteophyte formation [1], and majority of complaints about OA involve chronic pain, as well as losses of joint function. Approximately $1 \%$ of adult population in the world is affected by joint pain and disability resulting from ankle OA [2]. The incidence is increasing along with the population of elderly people and frequency of sports injuries [3-5]. It is considered that decrease of articular cartilage is related to the degree of ankle joint pain in patients with ankle OA. On the other hand, we considered radiographic ankle OA aren't equal to symptomatic ankle OA. Although therapeutic approach for relieving the pain associated with $\mathrm{OA}$ has been required, the mechanisms underlying the OA pain remain unclear. The reason is presumably due to the fact that experimental model of ankle OA has not been established.

Only age or surgically induced rat OA model is currently available [6]. The aging model has the obvious disadvantage of a longtime requirement and is associated with slight denaturation. The surgicalinduced model is made by cut of ligaments around the ankle joint. So it is dependent on the surgical skill of experimenters, which affects the degree of cartilage denaturation. We need to a lot of time to master the appropriate surgical procedure. This model is also associated with slight denaturation. We have difficulties in make ankle OA models uniformly in these methods.

On the other hand, a rat model of knee OA induced by the metabolic inhibitor monoiodoacetate (MIA) has been described both in terms of pathological disease progression [7] and more recently, pain related behavior [8-10]. Pomonis et al. compared MIA- and papain-induced knee OA models and found that only the MIA led to a chronic joint degeneration, indicated by decreases in bone mineral content and bone mineral density, as well as articular cartilage necrosis and osteophyte formation [11]. But we consider it is difficult to estimate pain related behaviors in these knee OA models because the knee isn't grounded. In addition, the knee joint is anatomically special in the joints of the body. The strong anterior and posterior cruciate ligaments are located only in the knee joint and provide stability, function. The rupture of these ligaments is often causing pain. We 
think the ankle OA model is more appropriate than knee OA model for the study about mechanisms of OA pain.

We therefore developed a new model of ankle OA rat induced by the application of MIA. We expected that an intra-articular MIA injection would initiate pain related behavior and histological OA-associated changes in the ankle joint. We confirmed the joint space in the ankle is narrower than that in the knee and the articular cartilage of the ankle is thicker than that of the knee in the rats from the preliminary experiments. Then we decided to inject MIA $(1 \mathrm{mg} / 30 \mu \mathrm{l})$ into the tibiotarsal joint at one time.

This study is to estimate the effects of intra-articular injection of MIA into the ankle on 2 consecutive days (MIA2 group) and 1 day (MIA1 group) relative to a saline-injected sham group. We examined with regards to pain related behaviors, ankle swelling and range of motion (ROM), gait effects, and pathological features in the three groups.

\section{Materials and Methods}

\section{Animal preparations and an intra-articular injection}

All experimental procedures were performed according to the Ethical Guidelines of the International Association for the Study of Pain and were approved by the Animal Care and Use Committee of Sapporo Medical University School of Medicine, Sapporo, Japan. Efforts were made to minimize the number of animals used.

Initially, we used 60 male Sprague-Dawley rats (3 weeks old; body weights: $46-58 \mathrm{~g})$. The rats were divided into three experimental groups of 20 rats ( 15 for behavioral study and 5 for pathological study). OA was induced by injecting MIA $(1 \mathrm{mg} / 30 \mu \mathrm{l})$ into the tibiotarsal joint of the right hind paw on 2 consecutive days (MIA2 group) or single day (MIA1 group). Sham rats treated via intra-articular saline injection on 2 consecutive days.

\section{Ankle measurements}

We examined the differences in the transverse and anteroposterior diameters and ROM of the affected and unaffected ankles at all stated time points. Ankle diameters were measured using digital calipers with the ankle at $90^{\circ}$ dorsiflexion (neutral position). We measured each diameter three times at all time points.

ROMs of the ankles were measured using a protractor. And we subtracted the dorsiflexion angle from the plantarflexion angle and calculated the arc on the right and left ankles. The differences between arc of the right and left ankles were compared at all time points.

We also measured the walking stride length on an acrylic board (7 $\mathrm{cm} \times 150 \mathrm{~cm} \times 12 \mathrm{~cm}$ ) at all stated time points. The stride length was measured three times using an iPad Air 2 device (Apple, Cupertino, CA, USA), and the average difference between the affected and unaffected sides were calculated.

\section{Behavioral analysis}

Pain related behavior was assessed by observing the mechanical and thermal hind paw withdrawal responses before injection (pre-MIA/ saline) and on days $7,14,21$, and 28 post-injection.

The mechanical sensitivity was evaluated as the frequency of withdrawal responses. Rats were placed in a Plexiglas chamber (IITC
Life Science Inc., Woodland Hills, CA, USA; $18 \mathrm{~cm} \times 25 \mathrm{~cm} \times 18 \mathrm{~cm}$ ) above a wire mesh floor, which allowed full access to the environment for at least $20 \mathrm{~min}$ before the test. Mechanical withdrawal responses to von Frey filament stimuli of $4 \mathrm{~g}$, and $6 \mathrm{~g}$ (Semmes Weinstein Monofilaments, North Coast Medical Inc., San Jose, CA, U.S.A) were evaluated at the above-described time points as previous reports $[12,13]$. Both the right and left hind paw were stimulated 30 times, with at least 10 min between each session. The mechanical withdrawal frequency was recorded by subtracting the number of responses on the left side from that on the right side.

To evaluate hyperalgesia, the thermal paw withdrawal response was measured using a radiant heat source (Tail Flick Analgesia Meter; IITC Life Science Inc.) at all above-described time points. The rats were placed in a Plexiglas chamber on a glass platform and allowed to acclimatize for at least $20 \mathrm{~min}$ before the test. The thermal withdrawal response was measured as the latency of hind paw withdrawal in response to thermal stimulation delivered by a radiant source that had been moved beneath the portion of the hind paw that was flush against the glass. Each hind paw was tested five times, and the mean withdrawal latency was calculated. Thermal paw withdrawal latency was defined as asymmetrical left-right latency.

\section{Histological assessments}

For the histological assessment, rats were sacrificed 28 days after MIA/saline injection, and the right ankle joints were dissected, fixed in $10 \%$ formalin, and embedded in paraffin. Sections were then stained with hematoxylin and eosin (HE) or safranin-O fast green. Histopathological changes on the distal medial/lateral tibia surface (DMT/DLT) and proximal medial/lateral talus surface (PMT/PLT) of the right hind paw tibiotarsal joint were observed via microscopy, and the severity of articular cartilage damage was evaluated using the Osteoarthritis Research Society International (OARSI) osteoarthritis cartilage histopathology assessment system [14]. In this system, the grade of damage from 0 (normal) to 6 (severe) is defined as the progression of OA into the cartilage and the stage of damage is defined as the horizontal extent of cartilage involvement from 0 (normal) to 4 (large). The final score is the combined value of grade and stage (score range 0 to 24 ).

\section{Statistical analysis}

All numerical data are expressed as means and standard errors of the means (SEM) and were analyzed using a one-way factorial measure of the analysis of variance (ANOVA) and the Tukey-Kramer test for comparisons among the three experimental groups. Student's $t$ test was used for analyses between two experimental groups. Data were considered statistically significant at $\mathrm{p}<0.05$.

\section{Results}

\section{MIA-induced ankle inflammation}

In both the MIA2 and MIA1 groups, significant increases in both the right ankle anteroposterior and transverse diameters (i.e., significant swelling) relative to the sham group were observed at all four post-injection time points (Figure 1A). Compared with the MIA1 group, the MIA2 group also exhibited significantly larger right ankle anteroposterior diameters on days 7 and 28 and significantly larger transverse diameters at all four post-injection time points (Figure 1B). 
Citation: Jimbo S, Terashima Y, Takebayashi T, Teramoto A, Ogon I, et al. (2017) A Novel Rat Model of Ankle Osteoarthritis Induced by the Application of Monoiodoacetate. J Arthritis 6: 260. doi:10.4172/2167-7921.1000260

Page 3 of 5

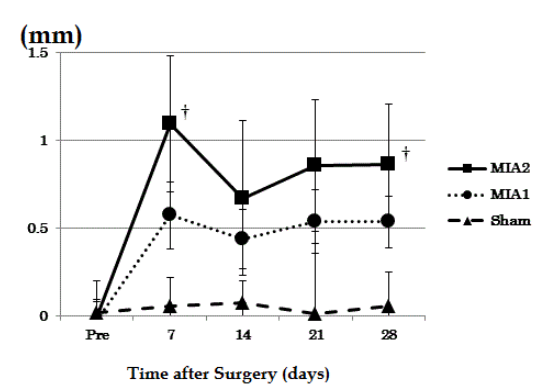

$(\mathrm{mm})$

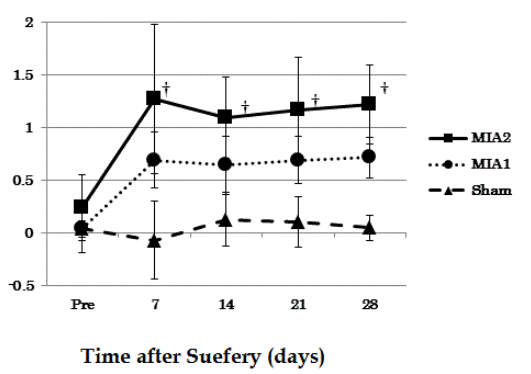

Figure 1: Ankle swelling. MIA injection increased the ankle anteroposterior (A) and transverse diameters (B) (i.e., swelling). Data are shown as the means \pm standard errors of the means. OA, osteoarthritis; MIA2, 2-day MIA injection protocol; MIA1, 1-day MIA protocol; Sham, 2-day saline injection. ${ }^{*} \mathrm{p}<0.05$ versus sham group; $\dagger \mathrm{p}<0.05$ versus MIA1 group.

\section{ROMs of the ankle and walking stride}

Both MIA groups exhibited significantly decrease ROMs of the ankles relative to the sham group at all four post-injection time points (Figure 2).

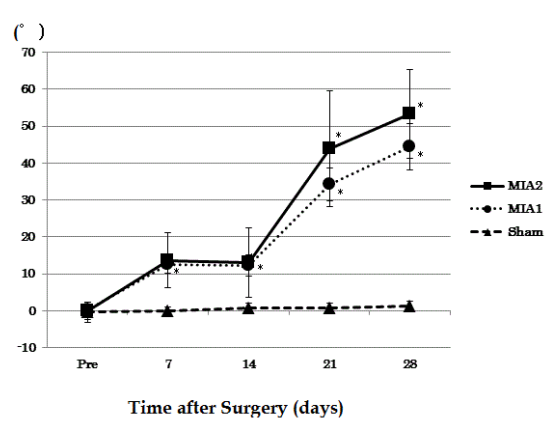

Figure 2: ROMs of the ankle (arc). MIA injection negatively affected range of motions of the ankle. Both MIA groups, the difference of ROM between the affected and unaffected side were increase. Data are shown as the means \pm standard errors of the means. OA, osteoarthritis; MIA2, 2-day MIA injection protocol; MIA1, 1-day MIA protocol; Sham, 2-day saline injection. ${ }^{*} \mathrm{p}<0.05$ versus sham group.
Furthermore, the MIA2 group exhibited a significant shorter walking stride relative to the sham group at all four times points, (Figure 3 ). The MIA1 group exhibited a significantly shorter stride relative to the sham group on days 21 and 28.

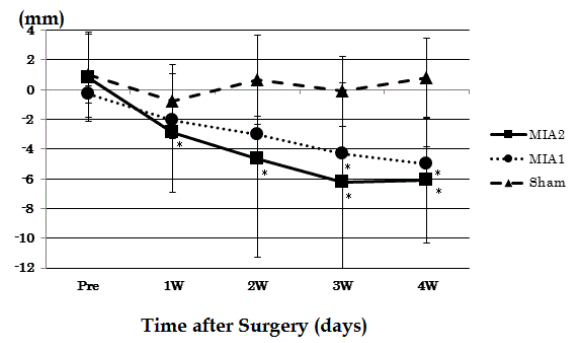

Figure 3: Stride length. MIA injection led to a shorter walking stride. Data are shown as the means \pm standard errors of the means. OA, osteoarthritis; MIA2, 2-day MIA injection protocol; MIA11day MIA protocol; Sham, 2 -day saline injection. ${ }^{*} \mathrm{p}<0.05$ versus sham group.

\section{Dose-specific effects of MIA on pain related behavior}

The mechanical response frequency was increased in the MIA2 group, compared with the sham group.
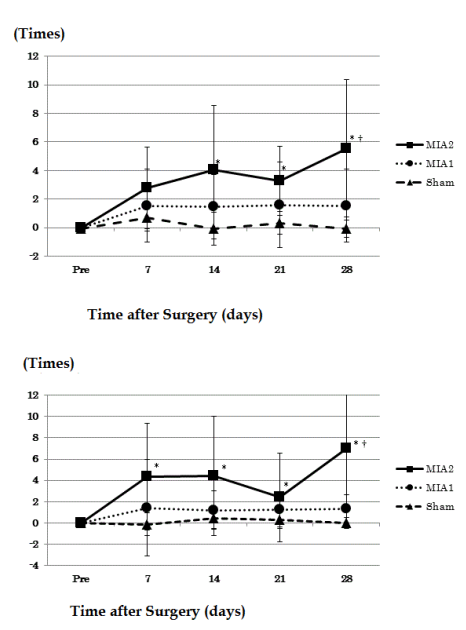

Figure 4: Assessment of mechanical hypersensitivity. Changes in pain-related behavior before and after MIA injection into the right hind paw. Mechanical allodynia was established in the osteoarthritis $(\mathrm{OA})$ and sham groups. No significant differences were observed between the MIA1 and sham groups. (A) von Frey 4-g, and (B) 6-g filaments. Data are shown as the means \pm standard errors of the means. OA, osteoarthritis; MIA2, 2-day MIA injection protocol; MIA1, 1-day MIA protocol; Sham, 2-day saline injection. ${ }^{*} \mathrm{p}<0.05$ versus sham group; $\uparrow \mathrm{p}<0.05$ versus MIA1 group.

Specifically, significant increases in the response frequency of the ipsilateral hind paw were observed; on days 14,21 , and 28 with the 4-g filament; and on all four post-injection days with the 6-g filament (Figure $4 \mathrm{~A}$ and $4 \mathrm{~B}$ ). In contrast, the MIA1 group did not differ 
Page 4 of 5

significantly from the sham group. Additionally, significantly shorter thermal latency was observed in the hind paws of both the MIA2 and MIA1 groups, compared with the sham group, on day 28 postinjection (Figure 5), whereas the MIA2 and MIA1 groups did not differ significantly.

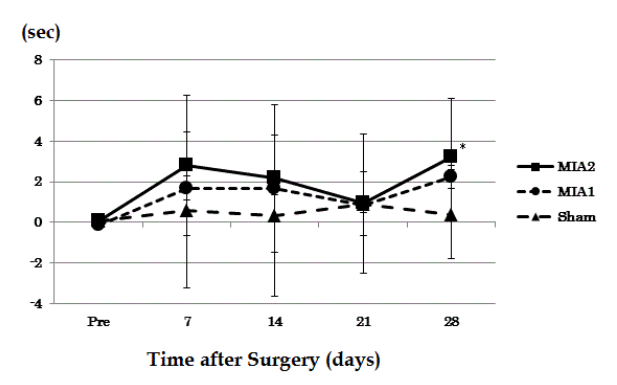

Figure 5: Assessment of thermal hyperalgesia. MIA injection caused thermal hyperalgesia in rats after 28 days. No significant difference was observed between the MIA2 and MIA1 groups. Data are shown as the means \pm standard errors of the means. OA, osteoarthritis; MIA2, 2-day MIA injection protocol; MIA1, 1-day MIA protocol; Sham, 2 -day saline injection. ${ }^{\star} \mathrm{p}<0.05$ versus sham group.

\section{Changes of cartilage and bone following the MIA injection}

Histological changes, such as chondrocyte disorganization, erosion, and cartilage surface fibrillation, and subchondral bone exposure, were observed in both MIA groups on day 28 , and were clearly dosedependent. The both MIA groups had significantly higher OARSI grade and stage except medial talus stage compared with sham rats. MIA2 rats had significantly higher OARSI stage in medial tibia compared with MIA1 rats. The both MIA groups had a significantly higher OARSI score in all parts on 28 days post-injection, compared with sham rats (Table1).

\begin{tabular}{|c|c|c|c|c|}
\hline & MIA2 & MIA1 & Sham & $\mathbf{P}$ \\
\hline \multicolumn{5}{|c|}{ Lateral tibia } \\
\hline Grade & $4.2(0.8)^{*}$ & $3.8(0.8)^{*}$ & $1.6(0.6)$ & 0 \\
\hline Stage & $3.4(0.6)^{*}$ & $2.6(0.6)^{*}$ & $1.2(0.5)$ & 0 \\
\hline Score & $14.6(5.1)^{*}$ & $10.2(4.0)^{*}$ & $1.8(0.5)$ & 0.001 \\
\hline \multicolumn{5}{|c|}{ Medial tibia } \\
\hline Grade & $4.4(0.6)^{*}$ & $3.2(0.6)^{*}$ & $1.2(0.5)$ & 0 \\
\hline Stage & $4.0(0)^{*} \dagger$ & $3.0(1.0)^{*}$ & $1.0(0)$ & 0 \\
\hline Score & $17.6(4.6)^{*} \dagger$ & $9.4(3.9)^{*}$ & $1.2(0.5)$ & 0 \\
\hline \multicolumn{5}{|c|}{ Lateral talus } \\
\hline Grade & $4.6(1.1)^{\star}$ & $3.2(0.8)^{*}$ & $1.4(0.6)$ & 0 \\
\hline Stage & $3.6(0.6)^{*}$ & $3.0(1.0)^{*}$ & $1.4(0.6)$ & 0.001 \\
\hline Score & $16.0(4.5)^{\star}$ & $11.8(6.0)^{\star}$ & $1.4(0.6)$ & 0.005 \\
\hline \multicolumn{5}{|c|}{ Medial talus } \\
\hline Grade & $4.6(1.1)^{\star}$ & $3.2(0.8)^{*}$ & $1.4(0.6)$ & 0 \\
\hline
\end{tabular}

\begin{tabular}{|c|c|c|c|c|}
\hline Stage & $3.0(0.7)$ & $2.8(0.8)$ & $2.2(0.8)$ & 0.292 \\
\hline Score & $13.6(3.9)^{\star}$ & $9.2(3.0)^{\star}$ & $1.5(1.2)$ & 0 \\
\hline
\end{tabular}

Data represent the means (standard error) for each group. Differences among the three groups were considered to be statistically significant when the $P$ value was under 0.05 by one-way analysis of variance and Tukey-Kramer test depending on normality of data. ${ }^{*} p<0.05$ versus sham group; $\uparrow p<0.05$ versus MIA1 group.

Table 1: Histopathologic evaluation of safranin-O fast green stained cartilage by OARSI osteoarthritis cartilage histopathology assessment system.

In addition, the MIA2 group exhibited a higher level of histological cartilage damage and abnormal medial tibia morphology, compared with the MIA1 group.

\section{Discussion}

$\mathrm{OA}$ is one of the most prevalent sources of chronic pain, and the increasing population age and high incidence of $\mathrm{OA}$ among the elderly suggests that a large number of patients suffer from OA-associated. In this study, we observed changes in ankle joints after the application of MIA. This is the first report to evaluate it. In the groups injected with MIA, osteophyte spike formation and synovial hyperplasia were observed in the tibia and talus of histology at 28 days after MIA injection. On the other hand, no such changes were seen in the group injected with saline at all days after saline injection. These results are similar to those of the previous study about the rat model of knee OA. MIA has been shown to inhibit chondrocyte metabolism and promote apoptosis [15], and its injection was found to induce various histological changes similar to those in human OA $[15,16]$. Kobayashi et al. [16] reported that an intra-articular injection of MIA induced OA-like histopathologic changes and a loss of proteoglycan from cartilage tissue in the knee joint in a dose-dependent manner. A MIAinduced model of knee OA was established and has been used in some studies $[7-10,15,16]$. We investigated the relationship between pain related behaviors and structural pathology of the ankle joint following MIA injection on single day or 2 consecutive days.

We note that the ankle joint space is small and cannot accommodate a large injection volume. Therefore, we generated our high-dose MIA model by injecting MIA on 2 consecutive days. Although both injection schedules led to significant difference in thermal hyperalgesia, ankle swelling, and ankle ROM relative to control rats, only the 2-day protocol led to increase hind paw mechanical hypersensitivity and a shorter stride. This result may indicate that peripheral sensitization have occurred only in the rats of the MIA 2 group.

Histologically, the MIA2 group also exhibited more degenerative articular cartilage changes (e.g., synovial thickening, loss of cartilage, osteophyte formation, talus collapse. Taken together, these data demonstrate that the 2-day MIA injection protocol is more suitable for generating a rat model of ankle OA.

The significant mechanical hypersensitivity (i.e. allodynia) observed in the MIA2 group, but not the MIA1 group, might indicate the presence of peripheral sensitization in the former group. On the other hand, the significant thermal hyperalgesia was observed in the MIA2 group only on day 28. It might indicate the presence of central sensitization at the time. Matthew et al. [10] further demonstrated the coexistence of increased central sensitization and referred pain, and 
Citation: Jimbo S, Terashima Y, Takebayashi T, Teramoto A, Ogon I, et al. (2017) A Novel Rat Model of Ankle Osteoarthritis Induced by the Application of Monoiodoacetate. J Arthritis 6: 260. doi:10.4172/2167-7921.1000260

Page 5 of 5

suggested the existence of a central change in $\mathrm{OA}$ similar to that observed with neuropathic pain. Zhang et al. [17] reported that OA pain correlated with peripheral and central sensitization, involvement of inflammatory cytokines, neuropeptides, and variety of chemical mediators. Hypersensitivity in OA has therefore been attributed to inflammation and degenerative changes within the ankle joint, as well as the excitability of intra-articular pain related receptors involving joint afferents.

A limitation of this study is that the ankle weight bearing of these rats is different from that of human. Further studies are needed in order to estimate the effect by weight of the rats.

\section{Conclusion}

We reported the new model of ankle OA, which is easily and rapidly generated, highly reproducible, and mimics human OA. We would like to clarify the mechanisms of occurrence and characteristics of OA pain by using this model hereafter. We will research the relationship between pain intensity and the changes of the cartilage and subchondral bone in the MIA2 and MIA1 groups by estimating the expression of pain producing substances. The results of the present study indicated OA pain might be related to peripheral sensitization or central sensitization which led to chronic pain. Further study is needed to reveal molecules that cause changes in the pain transmission in the nervous system. This would be of value for the treatment of OA pain.

\section{Conflicts of Interest}

The authors have no conflicts of interest with regard to this study.

\section{Acknowledgement}

No funds were received in support of this work.

\section{References}

1. Loeser RF, Goldring SR, Scanzello CR, Goldring MB (2012) Osteoarthritis: a disease of the joint as an organ. Arthritis Rheum 64: 1697-1707.

2. Cushnaghan J, Dieppe P (1991) Study of 500 patients with limb joint osteoarthritis. I. Analysis by age, sex, and distribution of symptomatic joint sites. Ann Rheum Dis 50: 8-13.

3. Kannus P, Palvane M, Niemi S, Parkkari J, Jarvinen M (2002) Increasing number and incidence of low-trauma ankle fractures in elderly people:
Finnish statistics during 1970-200 and projections for the future. Bone 31: 430-433.

4. Drawer S, Fuller CW (2001) Propensity for osteoarthritis and lower limb joint pain in retired professional soccer players. Br J Sportts Med 35: 402-408.

5. van Staa TP, Dennison EM, Leufkens HG, Cooper C (2001) Epidemiology of fractures in England and Wales. Bone 29: 517-522.

6. Chang SH, Yasui T, Taketomi S, Matsumoto T, Kim-Kaneyama JR, et al. (2016) Comparison of mouse and human ankles and establishment of mouse ankle osteoarthritis models by surgically-induced instability. Osteoarthritis Cartilage 24: 688-697.

7. Guzman RE, Evans MG, Bove S, Morenko B, Kilgore K (2003) Monoiodoacetate-induced histologic changes in subchondral bone and articular cartilage of rat femorotibial joints: an animal model of osteoarthritis. Toxicol Pathol 31: 619-624.

8. Fernihough J, Gentry C, Malcangio M, Fox A, Rediske J, et al. (2004) Pain related behavior in two models of osteoarthritis in the rat knee. Pain 112: 83-93.

9. Vonsy JL, Ghandehari J, Dickenson AH (2008) Differential analgesic effects of morphine and gabapentin on behavioural measures of pain and disability in a model of osteoarthritis pain in rat. Eur J Pain 13: 786-793.

10. Matthew T, Wahida R, Carl H, Anthony HD, David LB (2012) Characterisation of a Peripheral Neuropathic Component of the Rat Monoiodoacetate Model of Osteoarthritis. PLOS One 7: 1-11.

11. Pomonis JD, Boulet JM, Gottshall SL, Plillips S, Sellers R, et al. (2005) Development and pharmacological characterization of a rat model of osteoarthritis pain. Pain 114: 339-346.

12. Terashima Y, Kawamata M, Takebayashi T, Tanaka S, Tanimoto K, et al. (2011) Change in synaptic transmission of substantia gelatinosa neurons in a rat model of lumbar radicular pain revealed by in vivo patch-clamp recording. Pain 152: 1024-1032.

13. Miyakawa T, Terashima Y, Takebayashi T, Tanimoto K, Iwase T, et al. (2014) Transient receptor potential ankyrin 1 in spinal cord dorsal horn is involved in neuropathic pain in nerve root constriction rats. Mol Pain 10: 58.

14. Pritzker KP, Gay S, Jimenez SA, Ostergaard K, Pelletier JP, et al. (2006) Osteoarthritis cartilage histopathology: grading and staging. Osteoarthritis Cartilage 14: 13-29.

15. Kalbhen DA (1987) Chemical model of osteoarthritis-a pharmacological evaluation. J Reumatol 14: 130-131.

16. Kobayashi K, Imaizumi R, Sumichika H, Tanaka H, Goda M, et al. (2003) Sodium Iodoacetate-Induced Experimental Osteoarthritis and Associated Pain Model in Rat. J Vet Med Sci 65: 1195-1199.

17. Zhang RX, Ren K, Dubner R (2013) Osteoarthritis pain mechanisms: basic studies in animal models. Osteoarthritis Cartilage 21: 1308-1315. 